\title{
Reliability of ankle-brachial pressure index measured by pulse palpation method in diagnosing peripheral arterial disease among patients with diabetes mellitus
}

\author{
L D Ranasinghe ${ }^{1}$, N P Somasundaram ${ }^{1}$, S W A D A Wickramasinghe ${ }^{1}$, N Ranawake $^{1}$, K R C Jayasena ${ }^{1}$ \\ Sri Lanka Journal of Diabetes, Endocrinology and Metabolism 2015; 5: 65-68
}

\begin{abstract}
Introduction: Ankle brachial pressure index ( $\mathrm{ABI})$ is a useful screening test to detect peripheral arterial disease (PAD). However, the limitation for the widespread use of this test is the lack of doppler devices in most of the resource poor settings. In contrast, the pulse palpation method requires only a blood pressure apparatus with a suitable cuff and is a cheaper and readily available alternative.
\end{abstract}

Materials and methods: The objective of this study is to evaluate the accuracy and reproducibility of ABI measured by pulse palpation method and to study the correlation between the pulse palpation and doppler method. Population of 193 patients with diabetes were examined by two trained medical officers and $A B I$ was measured by each examiner using pulse palpation method $(p A B I)$ and doppler method $(d A B I)$.

Results: There was a statistically significant difference between the values obtained by the two observers for both $\mathrm{dABI}$ and $\mathrm{pABI}$. There was a significant difference between $\mathrm{dABI}$ and $\mathrm{pABI}$ measurements $(p<0.01)$. The $p A B I$ was lower than the $d A B I$, but there was a significant positive correlation between $\mathrm{dABI}$ and $\mathrm{pABI}$ in both lower limbs $(p<0.01)$.

Conclusion: According to our study, pABI had a sensitivity of $62 \%$ and a specificity of $90 \%$ in diagnosing PAD. Even though the doppler method cannot be replaced by pulse palpation method, there was a significant positive correlation between the two methods indicating that the $\mathrm{pABI}$ can be utilized to predict the $\mathrm{pABI}$ in resource poor setting.

Key words: ankle brachial pressure index, doppler device, peripheral arterial disease, resource poor setting, palpation method.

\section{Introduction}

PAD is common among patient with diabetes with a prevalence of $20 \%$ to $30 \%$ (4). It is also an important prognostic marker of cardiovascular disease related morbidity and mortality (1, 2, 3). Functional disability associated with PAD also leads to poor quality of life. PAD is usually severe and the outcome is poorer in patients with diabetes compared to patients without diabetes (5). Hence early detection and proper treatment of PAD in diabetic patients is of paramount importance in order to reduce the risk of cardiovascular events and longterm disability. Unfortunately, PAD is mostly silent and diagnosis is made at late stages.

$\mathrm{ABI}$ is a simple, non-invasive screening test, which can be used to diagnose as well as to assess the severity of PAD. The lower ABI values are associated with greater risk of cardiovascular events and the patients with the lowest $\mathrm{ABI}$ values have an annual mortality rate of about 25 percent $(6,7)$.
Due to the high prevalence of PAD in patients with diabetes, a screening ABI is recommended for all the patients with diabetes above the age of 50 years. When the PAD risk is high (when there are other PAD risk factors such as smoking, hypertension, hyperlipidemia or duration of diabetes $>10$ years) ABP is recommended even for the patients age less than 50 years (8). However, due to the unavailability of doppler devices and the lack of technical skills especially in resource poor setting, ABP is not frequently used in primary care setting for the screening of PAD.

ABI has a sensitivity of $90 \%$ and a specificity of $98 \%$ in detecting an angiographically defined stenosis of $\geq 50 \%$ (9). ABI values determined by simple pocket doppler devices can give similar results to that measured by automatic vascular laboratory equipment (10). ABI could also measured easily by pulse palpation method in primary care settings. However, previous studies done on accuracy of ABI measured by pulse palpation have not shown impressive results $(11,12)$.

${ }^{1}$ National Hospital of Sri Lanka. 
The objective of this study was to evaluate the accuracy and reproducibility of ABI measured by pulse palpation method in diagnosing PAD among patients with diabetes. Furthermore, we aimed to study the correlation between the pulse palpation and doppler method.

\section{Methods}

A cross sectional study was conducted at diabetes clinic in national hospital of Sri Lanka over a period of two months. Ethical clearance for this study was obtained from the ethical review committee of the national hospital of Sri Lanka.

Patients attending foot care clinic were included in the study after obtaining informed voluntary verbal consent. Patients who were having active ulceration or pain in legs which makes it difficult to measure ankle pressure and patients with past history of lower limb amputations were excluded. Those who did not consent were also excluded. Eligible patients were examined by two trained medical officers separately initially by palpation method followed by doppler method using hand held doppler device. Following a 10 minute resting period in supine position, systolic blood pressures (SBP) in the brachial, dorsal pedal and posterior tibial arteries were obtained by pulse palpation method and doppler method with a sphygmomanometer cuff placed $2 \mathrm{~cm}$ proximal to the malleoli or elbows. The first palpable or doppler impulse was used to identify the SBP at each location.

In doppler method, the pulses were located by palpation and the tip of the doppler probe was placed until an audible pulse signal is obtained. Then the pressure cuff was inflated $20 \mathrm{mmHg}$ above the point where the pulse is no longer audible. The cuff was slowly deflated at a rate of $2 \mathrm{mmHg}$ per second, noting the manometer reading at which the first pulse signal is heard and that was recorded as SBP. In pulse palpation method, pulse palpation was used instead of doppler signal to obtain SBP using the same technique. The ABI was calculated by dividing the highest systolic ankle pressure (either posterior tibial or dorsal pedal) in each leg by the highest systolic brachial pressure.

Data was analyzed using SPSS 17 software. Differences between measurements were assessed using one-sample Student's t-test. Multivariate linear regression analysis was used to assess the dependency of the observed difference between the two methods (correlation).

\section{Results}

We studied 193 patients. The means of the values obtained by observer A were; dABI left $1.08(\mathrm{SD}=0.17)$, $\mathrm{d} A B I$ right $1.07(\mathrm{SD}=0.15), \mathrm{pABI}$ left $1.01(\mathrm{SD}=0.14), \mathrm{pABI}$ right $1.03(\mathrm{SD}=0.13)$ whereas the means of the values obtained by observer B were; dABI left $1.11(\mathrm{SD}=0.18)$, dABI right $1.07(\mathrm{SD}=0.15), \mathrm{pABI}$ left $1.03(\mathrm{SD}=0.15)$, pABI right $1.03(\mathrm{SD}=0.16)$.

There was a statistically significant difference between the values obtained by observer $\mathrm{A}$ and $\mathrm{B}$ for both $\mathrm{dABI}$ and $\mathrm{pABI}$ of left lower $\operatorname{limb}(\mathrm{p}<0.01)$, indicating that there is a significant observer difference in obtaining the pulse and doppler values. Statistically significant differences were also observed between $\mathrm{dABI}$ and $\mathrm{pABI}$ $(\mathrm{p}<0.01)$ in both the observers A and B for both left and right lower limbs and $\mathrm{ABI}$ obtained from pulse palpation and Doppler method (palpation gave a lower value than that from doppler method).

Statistically significant correlations were observed between $\mathrm{dABI}$ and $\mathrm{pABI}$ in both the left and right lower limbs in A and B (figure 1) indicating that $\mathrm{AABI}$ can be predicted by pABI. Pulse palpation method had a sensitivity of $62 \%$ and a specificity of $90 \%$. The positive predictive value was $39 \%$ and the negative predictive value was $96 \%$.

In summary, there was a statistically significant difference between the values obtained by the two observers for both $\mathrm{dABI}$ and $\mathrm{pABI}(\mathrm{p}<0.01)$. There was a significant difference between $\mathrm{dABI}$ and $\mathrm{pABI}(\mathrm{p}<0.01)$ and $\mathrm{pABI}$ was lower than the $\mathrm{dABI}$. Interestingly, there was a significant positive correlation between $\mathrm{dABI}$ and $\mathrm{pABI}$ in both lower limbs $(\mathrm{p}<0.01)$. However, the sensitivity of pABI was only $62 \%$.

\section{Discussion}

The accuracy and reproducibility of the ABI can vary, according to the population studied, the cut-off threshold and the technique used to detect the blood flow in the arteries. According to available evidence, doppler method appears to be the most reliable method to determine the ABI. However, a recent meta-analysis of 8 studies of different populations, including patients with diabetes, showed a reasonably high specificity $(83 \%-99 \%)$ but a lower sensitivity (69\%-79\%) in detecting peripheral arterial disease (13). Several studies have reported even lower sensitivities (53\%-70\%) in diabetic patients (14). Our study has showed that a simple technique such as pulse palpation for ABP estimation can also have almost similar predictive values (specificity of $90 \%$ with a sensitivity of $62 \%$ )

Studies on intra observer and inter observer reproducibility of the $\mathrm{dABI}$ have shown varying results with intra observer coefficient of variation $(\mathrm{CoV})$ ranging from $4.7 \%$ to $13.0 \%(15,16)$. Our study also showed poor reproducibility of $\mathrm{dABI}$ among the observers. Hence, $\mathrm{ABI}$ appears to be highly operator dependent. Therefore, 

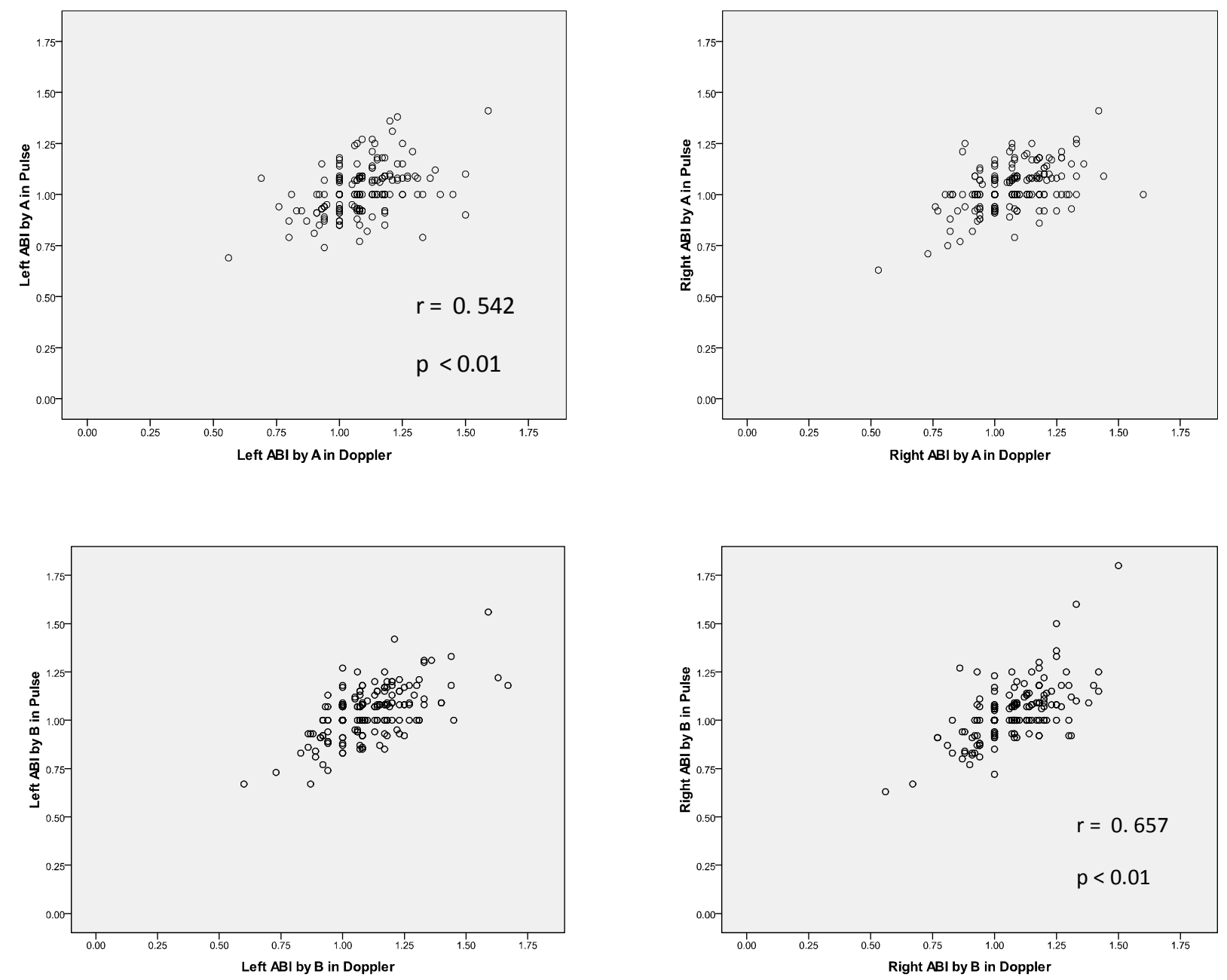

\section{Figure 1. Scatter plots with regression showing the correlation between $\mathrm{dABI}$ and $\mathrm{pABI}$.}

clinical judgment is important when interpreting the ABI results in patients at high risk of PAD. When clinical decisions are made, the possibility of falsely elevated ABI, especially in patients with diabetes and operator dependent variation of the results should be taken in to consideration. ABI immediately after treadmill exercise has shown to be more sensitive than resting $\mathrm{ABI}$, especially useful in patients who are on maintenance haemodialysis $(17,18)$ and it could be a worthy experiment to replicate this finding using palpation method.

Former studies have shown that pulse palpation method is inferior (sensitivity of $88 \%$ and a specificity ranging from $75 \%$ to $82 \%$ ) to the doppler method in detecting peripheral arterial disease $(12,19)$. However, our study showed different results with a sensitivity of $62 \%$ and a specificity of $90 \%$. Interestingly our study showed a significant positive correlation between $\mathrm{dABI}$ and $\mathrm{pABI}$ in both lower limbs $(\mathrm{p}<0.01)$ suggesting that this could be utilized as a useful alternative to doppler method in assessing peripheral arterial disease in resource poor setting.

\section{Conclusion}

$\mathrm{ABI}$ is a simple non-invasive measurement to detect PAD. Our study showed that both dABI and pABI are highly operator dependent. Therefore, ABI should be interpreted carefully in a given clinical context. Even though doppler method cannot be replaced by pulse palpation method, we noted a significant positive correlation between the two methods. This may indicate that the $\mathrm{dABI}$ can be predicted by $\mathrm{pABI}$ and $\mathrm{pABI}$ may be helpful in screening for PAD especially in resource poor setting.

\section{References}

1. Welten GM, Schouten O, Chonchol M, et al. Prognosis of patients with peripheral arterial disease. J Cardiovasc Surg 2009; 50(1): 109-21. 
2. Zheng ZJ, Sharrett AR, Chambless LE, et al. Associations of ankle-brachial index with clinical coronary heart disease, stroke and preclinical carotid and popliteal atherosclerosis: the Atherosclerosis Risk in Communities (ARIC) Study. Atherosclerosis 1997; 131(1): 115-26.

3. Resnick HE, Lindsay RS, McDermott MM, et al. Relationship of high and low ankle brachial index to allcause and cardio vascular disease mortality. Circulation 2004; 109(6): 733-9.

4. Elhadd TA, Robb R, Jung RT. et al. Pilot study of prevalence of asymptomatic peripheral arterial occlusive disease in patients with diabetes attending a hospital clinic. Practical Diabetes Int 1999; 16: 163-6.

5. Jude EB, Oyibo SO, Chalmers N, Boulton AJ. Peripheral arterial disease in diabetic and nondiabetic patients: a comparison of severity and outcome. Diabetes Care 2001; 24: $1433-7$

6. McKenna M, Wolfson S, Kuller L. The ratio of ankle and arm arterial pressure as an independent predictor of mortality. Atherosclerosis 1991; 87: 119-28.

7. Dormandy J, Heeck L, Vig S. The fate of patients with critical leg ischemia. Semin Vasc Surg 1999; 12: 142-7.

8. American Diabetes Association: Peripheral arterial disease in people with diabetes (Position Statement). Diabetes Care 2003; 26: 3333-41.

9. Yao ST, Hobbs JT, Irvine WT. Ankle systolic pressure measurements in arterial disease affecting the lower extremities. Br J Surg 1969; 56: 676-9.

10. Saskia PA, Nicolaï, Lotte M Kruidenier, et al. Pocket Doppler and vascular laboratory equipment yield comparable results for ankle brachial index measurement. BMC Cardiovasc Disord 2008; 8: 26.

11. Ena J, Lozano T, Verdú G, Argente CR, González VL.
Accuracy of ankle-brachial index obtained by automated blood pressure measuring devices in patients with diabetes mellitus. Diabetes Res Clin Pract 2011; 92(3): 329-36.

12. Aboyans V, Lacroix P, Doucet S, Preux PM, Criqui MH, Laskar M. Diagnosis of Peripheral Arterial Disease in General Practice: Can the Ankle-Brachial Index Be Measured Either by Pulse Palpation or an Automatic Blood Pressure Device. Int J Clin Pract 2008; 62(7): 1001-7.

13. Dachun X, Jue L, Liling Z, et al. Sensitivity and specificity of the ankle-brachial index to diagnose peripheral artery disease: a structured review. Vasc Med 2010; 15: 361-9.

14. Clairotte C, Retout S, Potier L, Roussel R, Escoubet B. Automated ankle-brachial pressure index measurement by clinical staff for peripheral arterial disease diagnosis in nondiabetic and diabetic patients. Diabetes Care 2009; 32: 1231-6.

15. Benchimol D, Pillois X, Benchimol A, et al. Accuracy of ankle-brachial index using an automatic blood pressure device to detect peripheral artery disease in preventive medicine. Arch Cardiovasc Dis 2009; 102: 519-24.

16. Stoffers HE, Kester AD, Kaiser V, Rinkens PE, Kitslaar PJ, Knottnerus JA. The diagnostic value of the measurement of the ankle-brachial systolic pressure index in primary health care. J Clin Epidemiol 1996; 49: 1401-5.

17. Hoogeveen EK, Mackaay AJ, Beks PJ, et al. Evaluation of the one-minute exercise test to detect peripheral arterial disease. Eur J Clin Invest 2008; 38(5): 290-5.

18. Kazuo Tsuyuki, Kenji Kohno, Kunio Ebine, et al. ExerciseAnkle Brachial Pressure Index with One-Minute Treadmill Walking in Patients on Maintenance Hemodialysis. Ann Vasc Dis 2013; 6(1): 52-6.

19. Migliacci R, Nasorri R, Ricciarini P, Gresele P. Anklebrachial index measured by palpation for the diagnosis of peripheral arterial disease. Fam Pract 2008; 25: 228-32. 